\title{
Coronal loop oscillations: energy considerations and initial value problem
}

\author{
J. Terradas ${ }^{\star}$, J. Andries ${ }^{\star \star}$, and M. Goossens \\ Centrum voor Plasma-Astrofysica, Katholieke Universiteit Leuven, Celestijnenlaan 200B, 3001 Leuven, Belgium \\ e-mail: jaume.terradas@wis.kuleuven.be
}

Received 5 March 2007 / Accepted 7 April 2007

\begin{abstract}
Context. Flares and eruptions in the solar corona generate oscillations of loops which have been interpreted as eigenmodes (mainly the fundamental kink mode, although other modes can also be excited). From the theoretical point of view the excitation of the tube eigenmodes due to an initial disturbance has not been studied in much detail.

Aims. The main aim of this work is to calculate for a given initial disturbance the amount of energy that is deposited in the trapped fast mode oscillation, how it depends on the initial perturbation and how it is distributed among the different eigenmodes (kink and fluting and also the longitudinal harmonics).

Methods. We calculate, using analytical expressions, the amplitude and the energy of the oscillation of the magnetic tube for different kinds of initial excitations.

Results. We find that external excitations deposit a small amount of energy in the tube. We show that fluting modes have quite small energies in comparison with the energy of the kink mode (around three orders of magnitude for the first fluting mode). On the contrary, the longitudinal fundamental mode and the longitudinal harmonics have energies of the same order of magnitude. In addition, we find that the loop length and density contrast can be important factors that determine the amount of energy that is trapped by the loop. Conclusions. The energy deposited in loops is typically six orders of magnitude smaller than the energy of the initial disturbance (for external excitations). However, it strongly depends on the distance of the initial perturbation and also on the loop properties (length and density). Fluting modes in coronal loops are very difficult to excite. Longitudinal harmonics are in principle more easily excited.
\end{abstract}

Key words. magnetohydrodynamics (MHD) - Sun: corona - Sun: magnetic fields - waves

\section{Introduction}

Several kinds of wave motions have been observed in coronal loops using the TRACE and SOHO satellites and other groundbased instruments. The observed loop oscillations (see for example Aschwanden et al. 2002; Schrijver et al. 2002) have been compared with the information of the theoretical models of coronal loop oscillations (see Roberts 2002, 2004). A possible interpretation of these oscillations is that they are the natural motions of the tube. With the use of the interpretation in terms of magnetohydrodynamic (MHD) eigenmodes, coronal seismology, suggested by e.g. Uchida (1970) and Roberts et al. (1984), has been performed by Nakariakov \& Ofman (2001) to determine the magnetic field and by Goossens et al. (2002); Aschwanden et al. (2003) and Arregui et al. (2007) to determine the density contrast and the density inhomogeneity length scale.

Although most of the TRACE observations suggest that the kink mode $(m=1)$ is excited since the loop axis is displaced laterally, other modes are probably also excited, for example the sausage and fluting modes. The sausage or pulsating modes are not trapped for thin and long loops but they can exist in fat and dense flare or post-flare loops. Nakariakov et al. (2003) and

^ Also associated at the Departament de Física, Universitat de les Illes Balears, 07122 Palma de Mallorca, Spain.

$\star \star$ Postdoctoral Fellow of the National Fund for Scientific ResearchFlanders (Belgium) (F.W.O.-Vlaanderen).
Aschwanden et al. (2004) have suggested that these modes, having short periods of the order of seconds, have been detected in the radio band. Fluting modes are trapped even in thin and long loops with a frequency quite similar to the frequency of the kink mode. However, up to now there is no observational evidence of such modes.

Additionally, observations indicate that the most excited mode is the fundamental mode in the longitudinal direction, i.e. the loop has a node at the footpoints and a maximum at half the loop length. There is only one example of excitation of the first longitudinal harmonic (Verwichte et al. 2004). This mode has a maximum at a quarter of the loop length and a node at half the loop length (in addition to the nodes at the footpoints). The period of the mode is basically half the period of the fundamental mode, however longitudinal density stratification can modify this ratio. The difference between the real ratio and 2 has been used by Andries et al. (2005) as a seismological tool to obtain information about the density scale height in loops.

Although the main features of the rich spectrum of eigenmodes in a coronal magnetic tube are well know, the problem of the excitation of the eigenmodes for a given initial perturbation has been only partially addressed (see Ruderman \& Roberts 2006; Terradas et al. 2006). In the present paper, this problem is investigated in detail and given an initial perturbation, the amount of energy that is distributed among the modes is calculated. We study how the energy of the trapped modes depends on 
the characteristics of the initial perturbation, namely the shape and location, and also on the loop properties. We focus on the energy that is trapped in the loop and not on the energy that is radiated away to wave leakage. This last issue has been investigated using straight loop models (see for example Cally 1986, 2003; Terradas et al. 2005, 2007; Pascoe et al. 2007) and also considering curved loops (Brady \& Arber 2005; Brady et al. 2006; Díaz et al. 2006; Selwa et al. 2006, 2007; Verwichte et al. 2006a-c).

This paper is organised as follows. In Sect. 2 a brief description of the tube eigenmodes is given and the energy associated with the different modes is introduced. In Sect. 3 we consider the initial value problem and we describe the method for calculating the energy trapped by the loop given an initial perturbation. The results for simple initial excitations are shown in Sect. 4. More complex perturbations are briefly studied in Sect. 5. In Sect. 6 the main conclusions are drawn.

\section{Tube eigenmodes and trapped wave energy in the cold plasma approximation}

The main properties of the loop eigenmodes using a straight cylindrical tube model have been studied by several authors (see for example Spruit 1981; Edwin \& Roberts 1983; Cally 1986, 2003). The dispersion diagram under coronal conditions and in the zero- $\beta$ limit indicates that only fast body waves are allowed. In the long-wavelength limit (i.e., when the tube radius is much smaller than the wavelength of the disturbance) the kink mode $(m=1)$ and the fluting modes $(m=2,3,4, \ldots)$ basically oscillate with the kink frequency. In contrast, the sausage mode $(m=0)$ is leaky at this limit and is only trapped for wavenumbers larger than a cut-off value (see Edwin \& Roberts 1983; Roberts et al. 1984).

The frequency of the modes, $\omega_{m}$, is determined by solving the dispersion relation which is derived by imposing continuity of the total pressure perturbation, $P$, and radial velocity, $v_{r}$, at the loop boundary $(r=R)$. The eigenfunction is written in terms of Bessel and Hankel functions $\left(J_{m}\right.$ and $\left.H_{m}^{(1)}\right)$. In particular, the total pressure perturbation is

$P(r, \varphi, z, t)= \begin{cases}\alpha \frac{J_{m}\left(\Lambda_{\mathrm{i}} r\right)}{J_{m}\left(\Lambda_{\mathrm{i}} R\right)} \sin (m \varphi) \sin (k z) \cos \left(\omega_{m} t\right), & 0 \leq r \leq R, \\ \alpha \frac{H_{m}^{(1)}\left(\Lambda_{\mathrm{e}} r\right)}{H_{m}^{(1)}\left(\Lambda_{\mathrm{e}} R\right)} \sin (m \varphi) \sin (k z) \cos \left(\omega_{m} t\right), & r>R,\end{cases}$

where

$\Lambda^{2}=\frac{\omega^{2}-\omega_{\mathrm{A}}^{2}}{v_{A}^{2}}$,

$\omega_{\mathrm{A}}=k v_{\mathrm{A}}$ is the Alfvén frequency, $k$ the longitudinal wavenumber and $v_{\mathrm{A}}$ the Alfvén speed. The integer $m$ denotes the azimuthal number of the mode and the subscripts $i$ and $e$ indicate that the magnitude has to be evaluated inside or outside the loop respectively. The factor denoted by $\alpha$ in Eq. (1) depends on the disturbance that leads to the excitation of the eigenmode. Given an initial perturbation the subsequent evolution of the tube can be written as a superposition of eigenmodes. Different initial conditions excite different eigenmodes with different amplitudes. The main aim of this paper is to investigate how the amplitude of the (non-leaky) eigenmodes depends on the initial perturbation and on the loop properties.

In order to compare different initial perturbations, all perturbations must represent the same amount of energy. The wave



Fig. 1. Energy density as a function of the radial position for the modes $m=1, m=2$ and $m=3$. In this plot the three modes have the same total energy $E$ (energy density integrated over space). The loop length is $L=50 R$ and the density contrast is $\rho_{\mathrm{i}} / \rho_{\mathrm{e}}=3$. The sausage mode is not represented since for these loop parameters this mode is not trapped.

energy density in the zero- $\beta$ approximation is (see for example Bray \& Loughhead 1974)

$e=\frac{1}{2}\left[\rho\left(v_{r}^{2}+v_{\varphi}^{2}\right)+\frac{1}{\mu}\left(b_{r}^{2}+b_{\varphi}^{2}+b_{z}^{2}\right)\right]$.

The first term corresponds to the kinetic energy (the internal energy and $v_{z}$ are zero due to the zero- $\beta$ limit) and the second term represents the magnetic energy. Note that Eq. (3) has to be integrated over space to represent the entire energy in the system. Since the initial perturbation can be viewed as a superposition of different eigenmodes of the system, it is useful to calculate, using the previous equation, the energy of the eigenmodes. Due to the temporal dependence of the eigenfunction, the kinetic and the magnetic energy density are out of phase in time for a fixed position. However, as expected, the total energy of the eigenmode (energy density integrated over space, denoted by $E$ ) is constant with time. This also means that there is energy equipartition between the total kinetic and magnetic energy. Figure 1 shows the behaviour of the energy density with the radial coordinate for different eigenmodes (normalised to the total energy of the eigenmode). The energy of the kink mode is rather uniformly distributed inside the tube. On the other hand, the energy density of the fluting modes has a peak at the loop boundary. This peak gets narrower when the order of the mode is increased (compare $m=2$ and $m=3)$. Note that outside the loop, $(r \gtrsim R)$ the kink mode has the largest energy.

From the observations we can estimate the amplitude $\alpha$ and the energy of the oscillating loops. According to the eigenfunction of the kink mode, the radial displacement at the loop radius $R$ is

$\begin{aligned} \xi(R, \varphi, z, t)= & \alpha \frac{1}{\omega_{m}^{2}-\omega_{\mathrm{Ae}}^{2}} \frac{\Lambda_{\mathrm{e}}}{\rho_{\mathrm{e}}} \frac{H_{m}^{(1)^{\prime}}\left(\Lambda_{\mathrm{e}} R\right)}{H_{m}^{(1)}\left(\Lambda_{\mathrm{e}} R\right)} \\ & \times \sin (m \varphi) \sin (k z) \sin \left(\omega_{m} t\right) .\end{aligned}$

Since for the kink mode $(m=1)$ the radial displacement is basically the transversal displacement of the loop axis, we can easily calculate the value of the amplitude $\alpha$. The observations provide a measure of the displacement of the loop, $\xi$, and we can compute the terms in the right hand side of the previous expression ( $\alpha$ is the unknown) using the frequency of the mode $\omega_{m}$ and the loop parameters. If an oscillating loop displaces the tube axis a distance equal to its radius then $\xi=R$. Let us assume that the loop parameters are $L=180 \mathrm{Mm}, R=4 \mathrm{Mm}, v_{\mathrm{Ai}}=10^{3} \mathrm{~km} \mathrm{~s}^{-1}$ 
$\rho_{\mathrm{i}} \approx 10^{-12} \mathrm{~kg} \mathrm{~m}^{-3}$ and $\rho_{\mathrm{e}} / \rho_{\mathrm{i}}=3$ (these are the typical values of the loops that show oscillations). Using these values in Eq. (4) we find that $\alpha=2.42 \times 10^{-3}$ (we have dropped the spatial and temporal harmonic dependences). Once the amplitude is known the energy of the loop is calculated with the integral over space of the energy density (Eq. (3)) using the eigenfunction. We eventually find that $E \sim 10^{19} \mathrm{~J}$. This is the total energy of the kink mode inferred from the observations and using the interpretation in terms of the eigenmode of the loop.

\section{Initial value problem}

We now turn to the theoretical study of how the amplitude of oscillation (i.e. the amplitude $\alpha$ introduced in the previous section) depends on the initial disturbance. There are several ways to study the initial value problem. The first method is to solve the time-dependent problem numerically and to derive from the results the amount of energy that is deposited in the normal modes of the structure. The advantage here is that the behaviour of the tube is known at any time. Another method is to use analytical approximations of the solution. This has been done recently by Ruderman \& Roberts (2006) who solved the initial value problem using the Laplace transform to determine the motion of the tube. As expected, these authors have found that the asymptotic behaviour of the loop for $t \rightarrow \infty$ is given by the fundamental normal mode of the structure. Since we are mostly interested in the energy that is trapped in the loop and not in the transients before the loop settles in the normal mode, we will use the second approach.

Ruderman \& Roberts (2006) concentrated on the kink oscillation $(m=1)$. Here we have extended the results to $m \geq 1$. These authors studied initial perturbations in the total pressure given by the function $F$

$F=\frac{\mathrm{i} \omega P_{0}-\partial P_{0} / \partial t}{v_{\mathrm{A}}^{2}}$,

$P_{0}$ is the form of the total pressure perturbation at $t=0$ that depends on the coordinate $r$. Since there is no gas pressure the initial excitation is a perturbation in the longitudinal magnetic field $\left(b_{z 0}=P_{0} \mu / B_{0}\right)$. In addition, Ruderman \& Roberts (2006) considered the case with zero initial radial velocity (at $t=0$ ), i.e. $v_{r 0}=0$ and also that $b_{r 0}=0$. For simplicity we also assume that $\partial P_{0} / \partial t=0$, which means (according to the induction equation) that the azimuthal velocity perturbation is $v_{\varphi 0}=0$. Since $b_{r 0}=0$ and the initial disturbance must satisfy $\nabla \cdot \boldsymbol{b}_{0}=0$ this implies that the azimuthal component of the magnetic field disturbance must be (dropping the dependences with $z$ )

$b_{\varphi 0}=r \frac{k}{m} b_{z 0}$.

Therefore, the initial perturbation is a magnetic perturbation (in the longitudinal and azimuthal components) with zero initial velocity. This initial excitation is unable to excite sausage modes since the radial component of the magnetic field is zero and the divergence-free condition for the magnetic field cannot be satisfied when $m=0$. Other initial conditions are required to study the sausage mode.

The initial disturbance excites fast waves that propagate towards and away from the loop. Part of the energy is trapped by the density enhancement while most of the energy does not reach the loop or is eventually radiated from the tube (through the leaky modes). Ruderman \& Roberts (2006) showed that for $t \rightarrow \infty$ the tube is oscillating with the eigenmode, representing the trapped energy. Since the tube oscillates with the eigenmode the magnetic pressure is simply given by Eq. (1). An interesting result of the work of Ruderman \& Roberts (2006) is that an expression for the amplitude $\alpha$ is explicitly derived (they use $\chi_{m}$ as the amplitude, in our notation $\left.\alpha=2\left|\chi_{m}\right|\right)$. The coefficient $\chi_{m}$ depends on the particular form of the initial perturbation and it is given by

$\chi_{m}=\left.\frac{T}{\frac{\mathrm{d} D}{\mathrm{~d} \omega}}\right|_{\omega=\omega_{m}}$,

where

$$
\begin{aligned}
T(\omega)= & \Lambda_{\mathrm{i}}^{-1} \Lambda_{\mathrm{e}}^{3} H_{m}^{(1)}\left(\Lambda_{\mathrm{e}} R\right) \int_{0}^{R} s J_{m}\left(\Lambda_{\mathrm{i}} s\right) F_{\mathrm{i}}(s) \mathrm{d} s \\
& +\Lambda_{\mathrm{i}} \Lambda_{\mathrm{e}} J_{m}\left(\Lambda_{\mathrm{i}} R\right) \int_{R}^{\infty} s H_{m}^{(1)}\left(\Lambda_{\mathrm{e}} s\right) F_{\mathrm{e}}(s) \mathrm{d} s,
\end{aligned}
$$

and

$D(\omega)=\Lambda_{\mathrm{e}}^{2} \Lambda_{\mathrm{i}} R J_{m}\left(\Lambda_{\mathrm{i}} R\right) H_{m}^{(1)^{\prime}}\left(\Lambda_{\mathrm{e}} R\right)-\Lambda_{\mathrm{e}}^{3} R J_{m}^{\prime}\left(\Lambda_{\mathrm{i}} R\right) H_{m}^{(1)}\left(\Lambda_{\mathrm{e}} R\right)$.

The term $T$ is the convolution of the magnetic pressure disturbance, represented by $F$ (see Eq. (5)), with the magnetic pressure eigenfunction (Eq. (1)). Notice the expected distinction between the contribution of the internal (first term of Eq. (8)) and external part of the tube (second term). The coefficient $D$ represents the dispersion function, i.e. the zeros of this function correspond to the frequencies of the normal modes.

\subsection{General form of the initial perturbation}

In the previous analysis the amplitude $\alpha$ and consequently also the energy deposited in a mode with fixed wavenumbers $k$ and $m$ has been calculated. However, in general a real perturbation is not restricted to a single $k$ and $m$ pair, but it requires a sum over several different wavenumbers. If the form of the initial perturbation in the azimuthal and vertical direction is $f(\varphi, z)$ then we can write

$f(\varphi, z)=\sum_{m=1}^{\infty} \sum_{n=1}^{\infty} a_{m, n} \sin m \varphi \sin (\pi n z / L)$,

where

$a_{m, n}=\frac{4}{\pi L} \int_{0}^{\pi} \int_{0}^{L} f(\varphi, z) \sin m \varphi \sin (\pi n z / L) \mathrm{d} \varphi \mathrm{d} z$.

Thus, the function is decomposed in Fourier sinus series in the azimuthal direction (we are assuming that the initial perturbation is odd in the interval $-\pi<\varphi<\pi$, and thus the $m=0$ contribution is zero) and in sinus series in the longitudinal direction (note that the function must be zero at $z=0$ and at $z=L$ ). For any $m$ and $n$ in the decomposition we can calculate from the dispersion relation the frequency $\omega_{m, n}$ and then we can evaluate the corresponding amplitude $\alpha_{m, n}$. Thus, for example, the pressure perturbation at the loop radius will be now given by

$P(R, \varphi, z, t)=\sum_{m=1}^{\infty} \sum_{n=1}^{\infty} \alpha_{m, n} \sin m \varphi \sin (\pi n z / L) \sin \left(\omega_{m} t\right)$.

It is straight-forward to calculate the displacement and energy. 


\subsection{Method}

From the practical point of view, for a given form of the initial perturbation, $P_{0}$, we determine the corresponding decomposition as a sum of azimuthal numbers $m$ and longitudinal wavenumbers $k_{n}=n \pi / L$. By evaluating Eq. (7) the amplitude $\left(\alpha_{m, n}=2\left|\chi_{m, n}\right|\right)$ associated with each pair of wavenumbers is calculated. First, we solve the dispersion relation and compute the eigenfrequency $\omega_{m, n}$. Second, the derivative of the dispersion function is calculated and it is evaluated at $\omega_{m, n}$. Finally, we calculate numerically the integral in the coefficient $T\left(\omega_{m, n}\right)$. Additionally, the energy of the mode is computed. This process is repeated for each set of values $m$ and $n$.

\section{Simple excitation: single modes}

The aim of this section is to investigate how the energy deposited in the eigenmodes depends on the properties of the initial disturbance and the properties of the loop. We first study in detail simple perturbations. More general excitations are addressed in the following section.

The initial perturbations that we study in this section are simple, in the sense that they can be represented by a single $k$ and $m$. Let us consider first the kink mode $(m=1)$ and the first fluting mode $(m=2)$ and concentrate on the fundamental longitudinal wavenumber $k=\pi / L$. For simplicity, we take the perturbation (in the magnetic pressure) to have the following form in the radial direction:

$P_{0}=P_{\mathrm{N}} \mathrm{e}^{-\left(\frac{r-r_{0}}{a}\right)^{2}}$,

where $P_{\mathrm{N}}$ is a normalisation constant, $r_{0}$ is the location of the Gaussian and $a$ is the width at half height. This allows us to study the behaviour of the loop for different locations of the disturbance and different widths of the initial pulse. The profile of the initial perturbation for $m=1$ and $m=2$ using the radial dependence given by Eq. (13) is displayed in Fig. 2. Obviously, they are not a good representation of disturbances produced by for example, a flare or eruption, however their analysis is necessary for the understanding of more realistic excitations.

In order to compare results for perturbations located at different positions we need to choose the normalisation constant, $P_{\mathrm{N}}$, in such a way that the energy of the initial perturbations is the same. For the perturbation given by Eq. (13), since $v_{r 0}=v_{\varphi 0}=$ $b_{r 0}=0, b_{z 0}=P_{0} \mu / B_{0}$ and $b_{\varphi 0}=r k / m b_{z 0}$, the total energy is

$$
\begin{aligned}
E_{0} & =\pi \frac{L}{4} \frac{\mu}{B_{0}^{2}} \int_{0}^{\infty} P_{0}^{2}\left(1+r^{2} \frac{k^{2}}{m^{2}}\right) r \mathrm{~d} r \\
& =\pi \frac{L}{4} \frac{\mu}{B_{0}^{2}} P_{\mathrm{N}}^{2} \int_{0}^{\infty} \mathrm{e}^{-2\left(\frac{r-r_{0}}{a}\right)^{2}}\left(1+r^{2} \frac{k^{2}}{m^{2}}\right) r \mathrm{~d} r .
\end{aligned}
$$

Thus, to keep the energy of the initial disturbance constant the following normalisation factor is defined,

$$
\begin{aligned}
P_{\mathrm{N}}^{2}= & E_{0} \frac{4 B_{0}^{2}}{\pi L \mu}\left[\int_{0}^{\infty} \mathrm{e}^{-2\left(\frac{r-r_{0}}{a}\right)^{2}}\left(1+r^{2} \frac{k^{2}}{m^{2}}\right) r \mathrm{~d} r\right]^{-1} \\
= & E_{0} \frac{4 B_{0}^{2}}{\pi L \mu} 16 m^{2}\left(a \left[2 a \mathrm{e}^{-2\left(\frac{r_{0}}{a}\right)^{2}}\left\{2 m^{2}+k^{2}\left(a^{2}+2 r_{0}^{2}\right)\right\}\right.\right. \\
& \left.\left.+\sqrt{2 \pi} r_{0}\left\{4 m^{2}+k^{2}\left(3 a^{2}+4 r_{0}^{2}\right)\right\}\left(1+\operatorname{Erf}\left(\frac{\sqrt{2} r_{0}}{a}\right)\right)\right]\right)^{-1},
\end{aligned}
$$

$\operatorname{Erf}$ is the error function. The normalisation function decreases with $r_{0}$ and $a$, and in the limit $k r_{0} / m \gg 1$, i.e. basically for

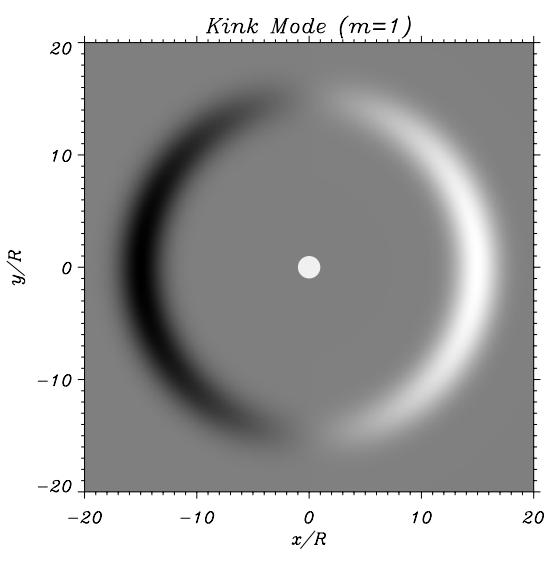

a)



b)

Fig. 2. a) Example of kink perturbation and b) fluting $(m=2)$ perturbation in the perpendicular plane to the loop axis. For this initial disturbance $r_{0}=15 R$ and $a=2 R$. In the vertical direction the dependence is $\sin (k z)$. White colour corresponds to positive magnetic pressure perturbations while black colour represents negative values. The circular white region in the centre represents the loop.

perturbations located at a distance much longer than the loop length $(k=\pi / L)$, is $P_{\mathrm{N}} \sim 1 / \sqrt{a r_{0}^{3}}$.

\section{1. $r_{0}$ and $\alpha$ dependence}

We have focused on perturbations located in the coronal medium $\left(r_{0} \gg R+a\right)$ and we have studied how the displacement at the loop boundary and the total energy change with $r_{0}$ and $a$. In Fig. 3 these two quantities are plotted for the $m=1$ mode. Both quantities decrease quite rapidly with $r_{0}$ (specially the energy) while they smoothly increase with the width of the initial perturbation $a$. This is a consequence of the convolution of the initial perturbation and the eigenfunction. From Eq. (7) we find that, since the contribution of the internal part of the loop (see Eq. (8)) is negligible due to the assumption $r_{0} \gg R+a$, the dependence with $r_{0}$ and $a$ is

$\alpha_{m} \sim P_{\mathrm{N}}\left(r_{0}, a\right) \int_{R}^{\infty} s H_{m}^{(1)}\left(\Lambda_{\mathrm{e}} s\right) \mathrm{e}^{-\left(\frac{s-r_{0}}{a}\right)^{2}} \mathrm{~d} s$.

As the distance of the perturbation increases the eigenfunction decreases and in consequence the integral is smaller. The normalisation constant also decreases with $r_{0}$ to keep the energy of the initial perturbation constant. Thus, the combination of these two tendencies results in a decrease of the amplitude with $r_{0}$. On the other hand, for a fixed $r_{0}$ when $a$ is increased, the integrals increase since the perturbation is wider (although $P_{\mathrm{N}}$ decreases 

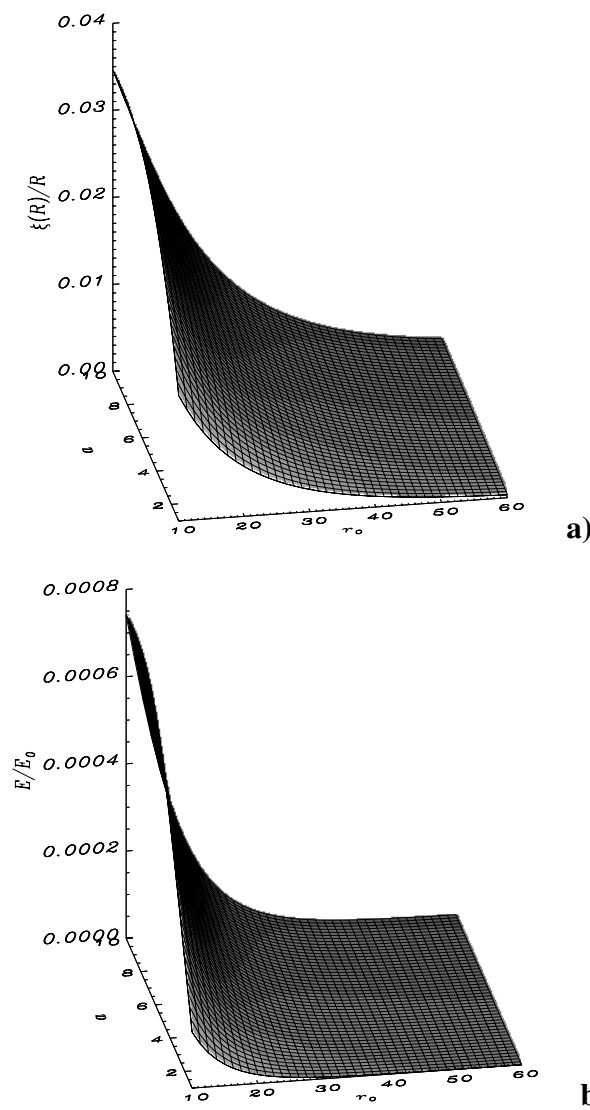

b)

Fig. 3. a) Displacement and b) trapped energy as a function of $r_{0}$ and $a$ for the $m=1$ mode. For these plots $L=50 R$ and $\rho_{\mathrm{i}} / \rho_{\mathrm{e}}=3$. The behaviour of the displacement and energy with $r_{0}$ and $a$ is given by Eqs. (18) and (19) respectively in the limit $r_{0} \gg R+a$.

with $a$, the dominant behaviour is due to the integral). In fact, it is possible to find the asymptotic behaviour with $r_{0}$ and $a$. Using the approximation for large arguments in the Hankel function $\left(H_{m}\left(\Lambda_{\mathrm{e}} r\right) \sim 1 / \sqrt{\left|\Lambda_{\mathrm{e}}\right| r} \mathrm{e}^{-\left|\Lambda_{\mathrm{e}}\right| r}\right)$ and assuming that $a \ll r_{0}$, we find that the integral is proportional to $\mathrm{e}^{-\left|\Lambda_{\mathrm{e}}\right| r_{0}} \sqrt{r_{0}} a$. Since $P_{\mathrm{N}} \sim \sqrt{a r_{0}^{3}}$ (valid for $k r_{0} / m \gg 1$ ) we eventually find that

$\alpha_{m} \sim \frac{\mathrm{e}^{-\left|\Lambda_{\mathrm{e}}\right| r_{0}}}{r_{0}} \sqrt{a}$.

The displacement has the same behaviour with $r_{0}$ and $a$ ( $\xi=$ $\left.\alpha_{m} \xi_{0}\right)$. Since the total energy is proportional to the square of the amplitude we simply have that

$E \sim \frac{\mathrm{e}^{-2\left|\Lambda_{\mathrm{e}}\right| r_{0}}}{r_{0}^{2}} a$.

These are the dependencies of the displacement and energy on $r_{0}$ and $a$ shown in Fig. 3.

Note that due to the strong dependence on $r_{0}$ in general only a small amount of the energy of the initial perturbation is trapped in the loop. For example, for a perturbation located at a distance similar to the loop length $r_{0}=40 R(L=50 R$ and $a=R)$, the trapped energy is $9.4 \times 10^{-7} E_{0}$.

We have done the same analysis for the first fluting mode ( $m=2$ ) and we have found that the behaviour with $r_{0}$ and $a$ is qualitatively similar to the results for the kink mode, specially in the limit of large $r_{0}$. Since we have normalised the energy of the initial pulse we can compare the trapped energy of these

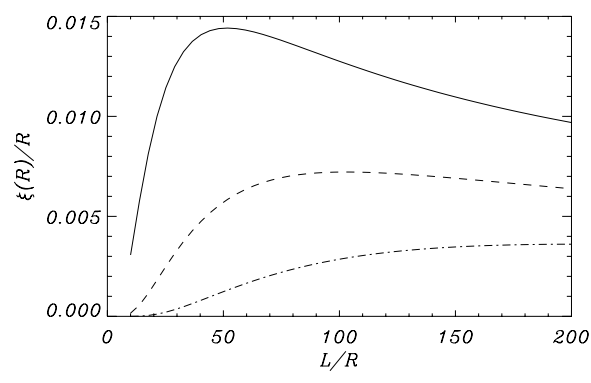

a)

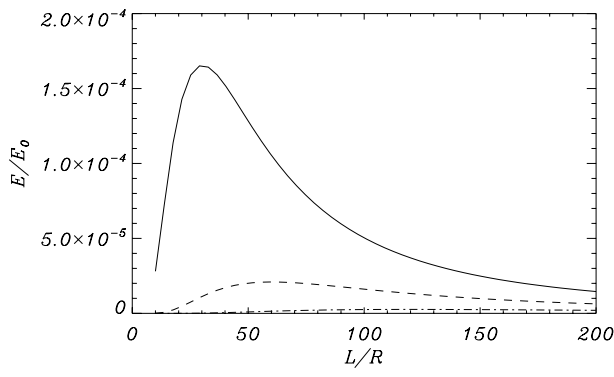

b)

Fig. 4. a) Displacement and b) energy as a function of the loop length. For all the cases the width of the perturbation is constant $a=R$. Solid line corresponds to $r_{0}=10 R$, dashed to $r_{0}=20 R$ and dotted-dashed corresponds to $r_{0}=40 R$. For these plots $\rho_{\mathrm{i}} / \rho_{\mathrm{e}}=3$.

two modes. The difference in energy between the modes is typically of three orders of magnitude. Therefore, fluting modes will be hardly excited in comparison with the kink modes. This is in accordance with Fig. 1.

\section{2. $L$ and $\rho_{i} / \rho_{e}$ dependence}

We now study the influence of the loop parameters on the amplitude of the displacement and the trapped energy. In particular, we investigate the dependence on the ratio between the length and the loop radius, $L / R$, and the density contrast between the tube and the coronal environment, $\rho_{\mathrm{i}} / \rho_{\mathrm{e}}$.

To study the effect of an initial perturbation on loops with different lengths we again normalise the initial perturbation using Eq. (16). Although the length of the system is now changed, the total energy of the initial perturbation is constant using this normalisation. In Fig. 4 the displacement and energy are plotted as a function of the loop length for different positions of the initial perturbation. There are several aspects of these curves that deserve comment. The displacement and energy have a maximum. This means that there are loops, with a preferred loop length $\left(L_{\max }\right)$, that are able to trap energy more efficiently than others. The position of this maximum depends on the location of the initial perturbation, and we see from Fig. 4 that $L_{\max }$ increases with $r_{0}$ (see the curves with different line styles). Note that the displacement and energy do not change much with the loop length for long loops $(L \geq 100 R)$. By contrast, short loops $(L \leq 50 R)$ are quite sensitive to the location of the initial perturbation.

The behaviour of the displacement as a function of $L$ in two different limits can be determined analytically. Using the thin tube approximation $(L \gg R)$ and the asymptotic behaviour of small arguments in the Bessel and Hankel functions (valid when $L \gg r_{0}$ ) we find that $\xi / R \sim 1 / \sqrt{L}$. This is the dependence present in Fig. 4a for $L \gg R$. On the contrary, for short loops in comparison with the loop radius the thin tube approximation is no longer valid, but using the approximation for large arguments 

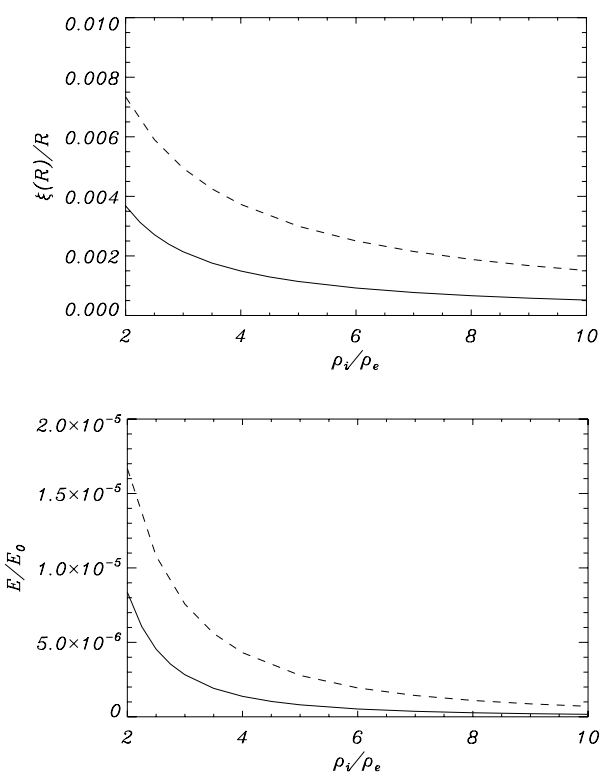

a)

b)

Fig. 5. a) Displacement at $r=R$ and b) energy as a function of the density contrast. The perturbation is located at $r_{0}=40 R, a=3 R$. The solid line corresponds to a loop with $L=50 R$ while the dashed line corresponds to $L=100 R$.

it is possible to show that $\xi / R \sim \mathrm{e}^{-\varepsilon / L}, \varepsilon$ being a constant. Since the displacement tends to zero in the two limits, the curve must show a maximum in between (see Fig. 4).

We have also studied how the results depend on the density contrast. Figure 5 shows the behaviour of the displacement and energy as a function of this parameter. Both the displacement and the energy decrease as $\rho_{\mathrm{i}} / \rho_{\mathrm{e}}$ is increased, which is the expected behaviour based on physical grounds. For the same initial perturbation high density contrast loops, i.e. basically heavy loops, are less excited than low density loops. We can see from Fig. 5a that, for example, loops with a density contrast of three oscillate, according to our model, with an amplitude that is three times larger than the amplitude of oscillation of loops with a density contrast of eight. Thus, the density contrast is another parameter that can have a strong influence on the amplitude of oscillation of loops. Note that the behaviour of the trapped energy with the density contrast is not in contradiction to the result that heavy loops radiate their energy more slowly than low density contrast loops (see for example Verwichte et al. 2006b; Selwa et al. 2007) due to the emission of leaky waves. This simply means that high density contrast loops are good waveguides because they have the eigenfunction very localised around the loop. But for the same reason, they are not very efficient in trapping energy from an external perturbation, since the trapped energy is proportional to the convolution between the initial perturbation and the eigenfunction.

\section{Two-dimensional perturbations}

In this section we investigate the extension of the method described before to more general and realistic perturbations. In particular, azimuthally and longitudinally localised perturbations are studied.



Fig. 6. Example of an azimuthally localised perturbation. The circular white region represents the loop. For this initial disturbance $r_{0}=15 R$, $a=2 R$ and $\delta=0.1$. This disturbance is decomposed in Fourier sinus series using Eq. (10).

\subsection{Azimuthally localised perturbation}

Let us assume that instead of single modes we have an external perturbation that has the same radial dependence as in Eq. (13) but now it has a Gaussian dependence on the $\varphi$ coordinate, i.e. we consider that the perturbation is

$P_{0}=P_{\mathrm{N}} \mathrm{e}^{-\left(\frac{r-r_{0}}{a}\right)^{2}} \mathrm{e}^{-\left(\frac{\varphi}{\delta}\right)^{2}} \sin (\varphi)$,

where $\delta$ is the width of the perturbation in the angular direction. Using the decomposition given by Eq. (11) (now $n$ is fixed) we calculate the total energy of the perturbation and we define the normalisation constant $P_{\mathrm{N}}$ accordingly. The sinus function has been included in the initial perturbation to ensure that there is no contribution of the $m=0$ mode. The shape of this initial perturbation is shown in Fig. 6 and it represents a more realistic disturbance than those studied in Sect. 4 (compare with the perturbations in Fig. 2).

It is simple to show that the energy of this initial disturbance is

$E_{0}=\sum_{m=1}^{\infty} E_{m}=\pi \frac{L}{4} \frac{\mu}{B_{0}^{2}} P_{\mathrm{N}}^{2} \sum_{m=1}^{\infty} a_{m}^{2} \int_{0}^{\infty} \mathrm{e}^{-2\left(\frac{r-r_{0}}{a}\right)^{2}}\left(1+r^{2} \frac{k^{2}}{m^{2}}\right) r \mathrm{~d} r,(21)$

where we have used the decomposition given by Eq. (10). The initial energy is written as the sum of the energy of the individual modes $\left(E_{m}\right)$. The normalisation constant, $P_{\mathrm{N}}$, is derived from this expression in a similar way as in the previous section.

We have studied a perturbation with $r_{0}=40 R, a=4 R$ and $\delta=0.5$. In Fig. 7a the energy of the modes in the angular decomposition is shown. For this particular perturbation the energy distribution in the initial perturbation decreases smoothly with the order of the harmonic $m$ (although the largest coefficient, $a_{m}$, in the Fourier decomposition of the pressure perturbation corresponds to $m=3$ ). The results of trapped energy associated with each $m$ are displayed In Fig. 7b. It is clear that the energy decreases with $m$ and that the kink mode has the largest energy. This behaviour is a direct consequence of the result that the energy trapped by the kink mode is between two and three orders of magnitude larger than the energy of the first fluting mode (see Sect. 4.1). Even for $m=1$ the trapped energy is quite small $\left(1.04 \times 10^{-6} E_{0}\right)$ and, as expected, is lower than that for an excitation with a single $m$ (we found for the same parameters that the energy was $3.77 \times 10^{-6} E_{0}$ ) since in that case all the initial energy was in one single mode. Qualitatively similar results are found 



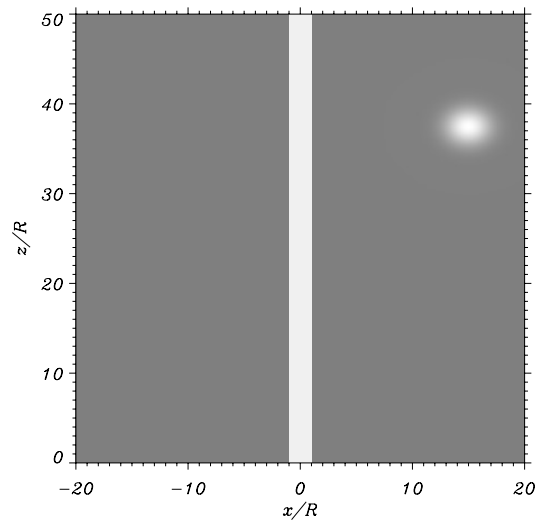

Fig. 8. Example of longitudinally localised perturbation. The white area represents the loop. For this initial disturbance $r_{0}=15 R, a=2 R, \Delta=$ $2 R$ and $z_{0}=L / 4$. This disturbance is decomposed in sinus series using Eq. (10) with $m$ fixed ( $m=1)$.

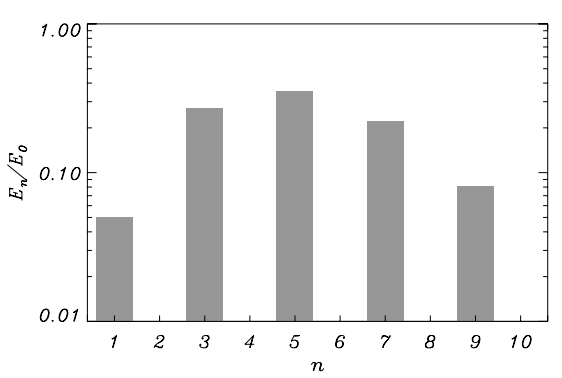

a)

when the width of the perturbation in the angular direction, $\delta$, is changed. Obviously, the energy of the modes in the initial perturbation is different, but again the trapped energy distribution is dominated by the kink mode.

\subsection{Longitudinally localised perturbation}

We can also study the energy distribution for a perturbation that has a Gaussian dependence with the $z$ coordinate,

$P_{0}=P_{\mathrm{N}} \mathrm{e}^{-\left(\frac{r-r_{0}}{a}\right)^{2}} \mathrm{e}^{-\left(\frac{z-z_{0}}{\Delta}\right)^{2}} \sin (z \pi / L)$,

where $z_{0}$ is the longitudinal location of the Gaussian and $\Delta$ is the width of the perturbation in the longitudinal direction. From the decomposition given by Eq. (11) (now $m$ is fixed) we calculate the total energy of the perturbation and derive the normalisation constant $P_{\mathrm{N}}$. The sinusoidal dependence on $z$ has been included in order to satisfy the line-tying condition. This perturbation is represented in Fig. 8.

Now the energy of the disturbance is simply

$E_{0}=\sum_{n=1}^{\infty} E_{n}=\pi \frac{L}{4} \frac{\mu}{B_{0}^{2}} P_{\mathrm{N}}^{2} \sum_{n=1}^{\infty} a_{n}^{2} \int_{0}^{\infty} \mathrm{e}^{-2\left(\frac{r-r_{0}}{a}\right)^{2}}\left(1+r^{2} \frac{k_{n}^{2}}{m^{2}}\right) r \mathrm{~d} r$,

where $k_{n}=n \pi / L$. Again this can be viewed as the sum of the energies of the individual modes of the decomposition in the vertical direction.

We have focused first on a perturbation with $m=1, r_{0}=$ $40 R, a=4 R, \Delta=5 R$ and located at half the loop length, $z_{0}=L / 2$. The energy of the modes in the initial perturbation is represented in Fig. 9a. Since the function is even in $z$ there is only a contribution from the odd $n\left(k_{n}=n \pi / L\right)$. Although the Fourier decomposition of this particular pressure perturbation is dominated by the mode with $n=2$, the most energetic is the $n=5$ (due to the dependence on $n$ in the previous equation). Figure $9 \mathrm{~b}$ shows that the fundamental mode is the most energetic and that the trapped energy decreases with the order of the

harmonic (the difference in energy between consecutive modes is typically of the order $10^{2}$ ).

We have also considered the case $z_{0}=L / 4$, i.e. a perturbation located at a quarter of the loop length. The energy decomposition of the initial perturbation is represented in Fig. 10a. Again the kink mode has the largest energy as can be appreciated in Fig. 10b. Nevertheless, the differences in energy between the longitudinal harmonics are not as large as for the $z_{0}=L / 2$ (now for example the fundamental and the first harmonic have energies of the same order of magnitude). This result also suggests that longitudinal harmonics are in principle more easily excited than azimuthal harmonics (with differences in the energy of the order $10^{3}$ ). 


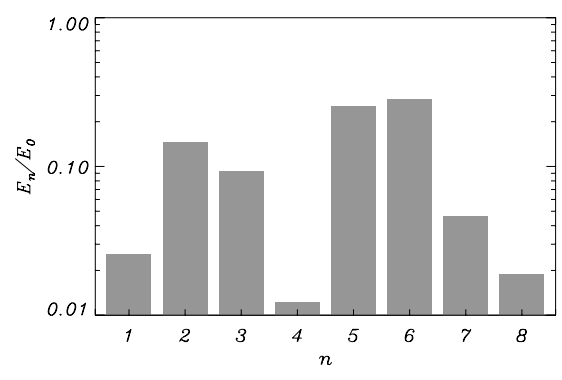

a)

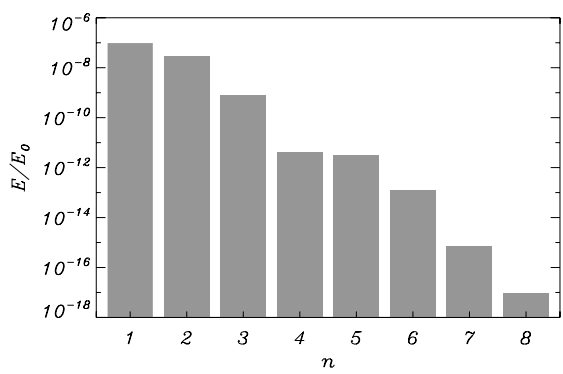

b)

Fig. 10. a) Energy of the Fourier components (calculated using Eq. (11) with $m$ fixed) of the initial perturbation given by Eq. (22). The $m=6$ has the largest coefficient in the decomposition. b) Trapped energy of each eigenmode (the vertical axis is in logarithmic scale). Note that the fundamental mode has the largest energy (although the first harmonic has the maximum weight in the decomposition). For this case $r_{0}=40 R$, $a=4 R(L=50 R), z_{0}=L / 4$ and $\Delta=L / 10$.

\section{Discussion and conclusions}

The energy that an initial disturbance stores in the eigenmodes of coronal loops has been examined. We have focused on external perturbations and we have found that when they are located far from the loop the trapped energy decreases quickly with the distance of the perturbation (as $\mathrm{e}^{-2\left|\Lambda_{\mathrm{e}}\right| r_{0}} / r_{0}^{2}$ ). Even for perturbations near the loop the trapped energy is several orders of magnitude smaller that the energy of the initial perturbation. This is mainly because first, not all of the energy of the initial perturbation reaches the tube and second, part of the energy does not get trapped in the loop (and it is probably radiated away through the excitation of the leaky modes). Therefore, most of the energy is in fact in the so-called "leaky continuum" (see Andries \& Goossens 2007) rather than in the eigenmodes representing the trapped energy. However, the estimate of the amount of energy in a particular mode can be a useful parameter that can give information about the possible damping mechanisms acting in the solar corona. Note that in the case of resonant absorption, this quantity is basically the energy that it is eventually dissipated and converted into heat.

According to our simple model, loops with different lengths and densities can respond quite differently to an initial perturbation. The loops with an optimal length are able to trap more efficiently the energy of the perturbation. On the other hand, short loops are less favourable for oscillations. Additionally, their density also plays a significant role in the wave trapping problem. We have found that for the same initial perturbation the trapped energy decreases quickly with the density contrast. These results suggest that these two quantities, i.e. the loop length and density contrast are important factors for the amplitude of oscillation in loops and can help to explain why, as reported from TRACE observations (Schrijver et al. 2002; Aschwanden et al. 2002), some loops oscillate clearly while other loops in the same active region, and even closer to the location of the perturbation, do not show any signature of oscillation.

For different kinds of disturbances we have found that the kink mode always traps the largest amount of energy in comparison with other modes. For example, the energy of the kink mode is typically $10^{3}$ larger than the energy of the first flute mode suggesting that these modes will rarely be excited for perturbations located far from the loop. This explains why it is justified to concentrate on the kink mode of oscillation since the contribution of the flute modes is rather small. Additionally, this could explain the fact that up to now no flute modes have been detected. However, other explanations are possible, for example that the motions of the fluting modes, displacing the loop boundary but not the loop axis, are not spatially resolved with the actual instruments. In contrast, the longitudinal harmonics are more easily excited. We have shown that for a perturbation localised close to one of the footpoints, the fundamental and first harmonic have energies of the same order of magnitude. Thus, from the observational point of view these modes are likely to be detected. In fact, there is an example of the detection of such a mode (see Verwichte et al. 2004; Andries et al. 2005).

There are estimations of the various types of energy released in real flares. However, it is not clear what amount of energy is associated with MHD waves. From the information of our model and the information of the coronal loop oscillations, this energy can be estimated. We have found that a typical oscillating loop has an energy of $10^{19} \mathrm{~J}$. If the perturbation is located at the loop length, then the energy of the initial disturbance should be $10^{6}$ times larger, i.e. of the order $10^{25} \mathrm{~J}$. This quantity is, in order of magnitude, similar to the estimations of the other types of energy. Thus, the energy of the oscillating loops can be potentially used as a seismological tool to determine the amount of energy released in real flares (related to fast MHD waves).

The model used here has several limitations. First, all the magnetic field lines have the same length, which is not realistic if we compare with the three-dimensional structure of bipolar regions. Second, we have studied the linear regime, however in a real flare a shock wave is produced and nonlinear effects are important and also couplings between the different harmonics are possible. An additional restriction of our study is that we have analysed only perturbations in the magnetic pressure, but in a real explosive event the initial disturbance can be produced for example by a density enhancement. In this regard, more general perturbations, especially those that are able to also excite the sausage modes need to be considered.

Acknowledgements. We thank the referee for comments which helped to improve this paper. J. Terradas is grateful to the K.U. Leuven (Department of Mathematics) for a fellowship. He also thanks the Spanish Ministry of Education and Science for the funding provided under the Juan de la Cierva program and for funding provided under grant AYA2006-07637.

\section{References}

Andries, J., Arregui, I., \& Goossens, M. 2005, ApJ, 624, L57

Andries, J., \& Goossens, M. 2007, Plasma Phys., 14, in press

Arregui, I., Andries, J., Van Doorsselaere, T., Goossens, M., \& Poedts, S. 2007, A\&A, 463, 333

Aschwanden, M. J., De Pontieu, B., Schrijver, C. J., \& Title, A. M. 2002, Sol. Phys., 206, 99

Aschwanden, M. J., Nightingale, R. W., Andries, J., Goossens, M., \& Van Doorsselaere, T. 2003, ApJ, 598, 1375

Aschwanden, M. J., Nakariakov, V. M., \& Melnikov, V. F. 2004, ApJ, 600, 458 
Brady, C. S., \& Arber, T. D. 2005, A\&A, 438, 733

Brady, C. S., Verwichte, E., \& Arber, T. D. 2006, A\&A, 449, 389

Bray, R. J., \& Loughhead, R. 1974, The Solar Chromosphere (ed. Chapmam)

Cally, P. S. 1986, Sol. Phys., 103, 277

Cally, P. S. 2003, Sol. Phys., 217, 95

Díaz, A. J., Zaqarashvili, T., \& Roberts, B. 2006, A\&A, 455, 709

Edwin, P. M., \& Roberts, B. 1983, Sol. Phys., 88, 179

Goossens, M., Andries, J., \& Aschwanden, M. J. 2002, A\&A, 394, L39

Nakariakov, V. M., \& Ofman, L. 2001, A\&A, 372, L53

Nakariakov, V. M., Melnikov, V. F., \& Reznikova, V. E. 2003, A\&A, 412, L7

Pascoe, D. J., Nakariakov, V. M., \& Arber, T. D. 2007, A\&A, 461, 1149

Roberts, B. 2002, Solar Variability: From Core to Outer Frontiers, ESA SP-506, 481

Roberts, B. 2004, Waves, Oscillations and Small-Scale Transient Events in the Solar Atmosphere: a Joint View from SOHO and TRACE, ESA SP-547, 1 Roberts, B., Edwin, P. M., \& Benz, A. O. 1984, ApJ, 279, 857
Ruderman, M. S., \& Roberts, B. 2006, J. Plasma Physics, 72, 285

Selwa, M., Solanki, S. K., Murawski, K., Wang, T. J., \& Shumlak, U. 2006, A\&A, 454, 653

Selwa, M., Murawski, K., Solanki, S. K., \& Wang, T. J. 2007, A\&A, 462, 1127 Schrijver, C. J., Aschwanden, M. J., \& Title, A. M. 2002, Sol. Phys., 206, 69 Spruit, H. C. 1982, Sol. Phys., 75, 3

Terradas, J., Oliver, R., \& Ballester, J. L. 2005, A\&A, 441, 371

Terradas, J., Oliver, R., \& Ballester, J. L. 2006, ApJ, 642, 540

Terradas, J., Andries, J., \& Goossens, M. 2007, Sol. Phys., accepted

Uchida, Y. 1970, PASJ, 22, 341

Verwichte, E., Nakariakov, V. M., Ofman, L., \& Deluca, E. E. 2004, Sol. Phys., 223, 77

Verwichte, E., Foullon, C., \& Nakariakov, V. M. 2006a, A\&A, 446, 1139

Verwichte, E., Foullon, C., \& Nakariakov, V. M. 2006b, A\&A, 449, 769

Verwichte, E., Foullon, C., \& Nakariakov, V. M. 2006c, A\&A, 452, 615 